\title{
Fractal behaviour of quantum paths in statistical physics
}

\author{
J.P.Badiali \\ Structure et Réactivité des Systémes Interfaciaux, \\ Université Pierre et Marie Curie, \\ 4 Place Jussieu, 75230 Paris Cedex 05, France
}

Received February 29, 2000

The path integral formalism is used to describe the statistical properties of an ideal gas of spinless particles. It is shown that the quantum paths exhibit the same properties in non-relativistic and relativistic domains provided the creation of new particles is avoided. Some quantities associated with the paths are introduced, they have a simple meaning if the quantity $\beta \hbar$, where $\beta$ is the reverse of the temperature, is considered as an ordinary time. The relation between the velocity on the path and the momentum is not the usual one, an extra term appears showing that the thermostat can not fix the average value of this velocity although all the thermodynamic quantities have their traditional values. The paths describe fluctuating trajectories on which the particles do not follow the equation of motion. For time intervals much shorter than $\beta \hbar$ we recover the properties of the Brownian motion. The trajectories are located in space in a volume restricted by the Compton wavelength for the short distances and the thermal de Broglie wavelength for the largest ones. It is shown that the time-energy uncertainty is verified on the quantum paths. This suggests that the density matrix obtained by quantification of the classical canonical distribution function via the path integral formalism should not be totally identical to that obtained via the usual route. Strong arguments are given showing that $\beta \hbar$ can be considered as an ordinary time and not as a formal quantity having the same dimension as time. This paper shows that for a time scale of 10 femtoseconds a totally new physics can be expected at room temperature. In addition it is suggested that the ratio $\hbar / k_{\mathrm{B}}$ may play a decisive role in the foundation of a covariant statistical physics.

Key words: path integral, statistical physics, fluctuations, relativity

PACS: 03.65.Ca, 05.30.-d, 05.70.-a, 47.53.+n

\section{Introduction}

It is a real pleasure for me to dedicate this paper to Professor I.R.Yukhnovskii for his 75 th anniversary. Due to him and to the scientists of his Institute, all my 
visits in Lviv were very fruitful and exciting in Sciences and also in many other respects.

Fractals and chaos represent two new frontiers in physics [1]. The concept of fractals has proven to be very useful to simulate irregular structures which can be self-similar or self-affine if different scalings have to be used for different variables. Fractals are associated with curves which are nowhere differentiable. The Brownian motion gives us an example of a random fractal [2]. Note that the term of Brownian motion is sometimes ambiguous since it may be associated with a trajectory in the plane (a self-similar curve) or with a trace (a self-affine curve) which represents the time history of spatial coordinates. To each one of these descriptions correspond different fractal dimensions [1]. In their seminal work which was published more than thirty years ago, Feynman and Hibbs [3] showed that the quantum paths exhibit a fractal character although the concept of fractal was not introduced at that time. Nowadays there is a tendency to consider the fractal geometry as a fundamental aspect of the physical world [4]. This point of view leads to two different options: we may assume that the particles obey a complicated dynamics which takes place in a simple space-time or, vice versa, that we have a simple dynamics though it takes place in a space-time having a complicated structure (a fractal structure, for instance). From a topological analysis of a fractal space-time some strong consequences can be derived as shown in [5].

In this paper, we would like to show that the fractal character of the quantum paths in quantum statistical physics is of a fundamental nature i.e. it is more than just a useful mathematical trick.

In what follows we start from the path integral formalism of statistical physics as given by Feynman [3] and we investigate some physical consequences of this formalism by considering a simple example, the ideal gas. In this case all the calculations can be performed analytically and the results are mathematically exact but their interpretation may be questionable. As we shall see these calculations lead to nontrivial results provided the time interval appearing in the formalism is considered as an ordinary time i.e. as a physical quantity that we can measure using a clock. Many aspects of this assumption will be analyzed.

The paper is organized as follows. In section 2 we recall some results obtained in [6]. Here, we insist on their physical meaning but not on their mathematical derivation which is rather simple. We investigate two specific properties of the paths: one is the mean square value of the velocity and the other one shows the correlation in the velocities. First derived in the non-relativistic case the results are extended to the relativistic domain. The consequences of relativistic dynamics are investigated while staying in the one-particle formalism. The properties of the paths are analyzed like in the non-relativistic case. In section 3 we discuss the physical meaning of the results. The results are first analyzed in terms of fluctuating trajectories and we consider the time-energy uncertainty relation associated with the paths. Then we reconsider the results by using some general arguments which are beyond the scope of the path integral formalism. In the last section the main conclusions of the paper are summarized. 


\section{Path integral formalism of statistical mechanics}

\subsection{The non-relativistic domain}

In the path integral formalism, the partition function, $Z$, can be written [7]

$$
Z=\frac{1}{(2 \pi \hbar)^{d}} \int D \mathbf{x}(t) \int D \mathbf{p}(t) \exp \left[-\frac{1}{\hbar} A\{\mathbf{x}(t), \mathbf{p}(t)\}\right]
$$

where $d$ is the dimension of space, $\mathbf{x}(t)$ and $\mathbf{p}(t)$ are the position and momentum vectors at time $t$ on a given trajectory, the symbols $D \mathbf{x}(t)$ and $D \mathbf{p}(t)$ mean that we have to perform a functional integration. In equation (1), $A\{\mathbf{x}(t), \mathbf{p}(t)\}$ is the Euclidean action. For the ideal gas, it is given by [7]:

$$
A\{\mathbf{x}(t), \mathbf{p}(t)\}=\int_{0}^{\beta \hbar} \mathrm{d} t\left[-\mathbf{i p}(t) \dot{\mathbf{x}}(t)+\frac{\mathbf{p}^{2}(t)}{2 m}\right],
$$

in which $m$ is the mass and $\beta$ the reverse of the absolute temperature $T, \beta=1 / k_{\mathrm{B}} T$. In the calculation of $Z$ we have to consider the cyclic paths for which $\mathbf{x}(0)=\mathbf{x}(\beta \hbar)$; this is reminiscent of the fact that the partition function is determined by the trace of the density matrix in standard quantum physics. In principle, in (1) there is an extra term related to the bosons statistics, we have dropped this term which is irrelevant in this work.

Using the usual procedure [7] the functional integral in (2) is transformed into a Riemann sum and the integrals in (1) will acquire a meaning in the limit $t_{n+1}-t_{n}=$ $\Delta t \rightarrow 0$ where $t_{n}$ and $t_{n+1}$ are two successive values of $t$ such as $0 \leqslant t_{n}<t_{n+1} \leqslant \beta \hbar$. In addition to the position and momentum variables a third quantity $\dot{\mathbf{x}}(t)$ appears in equation (2), it is defined according to $\dot{\mathbf{x}}(t)=\left(\mathbf{x}\left(t_{n+1}\right)-\mathbf{x}\left(t_{n}\right)\right) / \Delta t$, it can be considered as the velocity on the path. However, it is important to note that $\dot{\mathbf{x}}\left(t_{n}\right)$ is disconnected from the set of values $\left\{\mathbf{p}\left(t_{i}\right)\right\}$ taken by the momentum. Accordingly, on the quantum paths, no simple relation is expected between velocity and momentum.

If we consider some properties only related to the momentum we can perform the integration over the positions $\mathbf{x}(t)$ and we get the well known form of the partition function:

$$
Z=\frac{V}{(2 \pi \hbar)^{d}} \int \mathrm{d} \mathbf{p} \exp \left[-\beta \frac{\mathbf{p}^{2}}{2 m}\right]
$$

where $V$ is the volume of the sample. According to the equation (3) any function of the momentum will have the same value as in the classical case, in particular $\left\langle\mathbf{p}^{2}\right\rangle / 2 m=d /(2 \beta)$. From (3) we can see how the momenta are distributed at the thermal equilibrium. However, from (3) we have no information about the distribution of velocities. This can be illustrated by considering the velocity on the path. After a partial integration over the momentum we get

$$
Z=\frac{1}{(2 \pi \hbar)^{d}} A \int D \mathbf{x}(t) \exp \left[-\frac{1}{\hbar} \int_{0}^{\beta \hbar} \mathrm{d} t\left(\frac{1}{2} m[\dot{\mathbf{x}}(t)]^{2}\right)\right]
$$


where $\mathrm{A}$ is a well known normalization constant [3]. Instead of $Z$ we may define a more general quantity defined according to

$$
K\left(\mathbf{x}_{b}-\mathbf{x}_{a} ; t_{b}-t_{a}\right)=\frac{1}{(2 \pi \hbar)^{d}} A \int D \mathbf{x}(t) \exp \left[-\frac{1}{\hbar} \int_{t_{a}}^{t_{b}} \mathrm{~d} t\left(\frac{1}{2} m[\dot{\mathbf{x}}(t)]^{2}\right)\right]
$$

in which the position $x_{b}$ and $x_{a}$ associated with the times $t_{a}$ and $t_{b}$ respectively, are different. A more explicit form of $K\left(\mathbf{x}_{b}-\mathbf{x}_{a} ; t_{b}-t_{a}\right)$ can be obtained, we have [7]:

$$
K\left(\mathbf{x}_{b}-\mathbf{x}_{a} ; t_{b}-t_{a}\right)=\left(\frac{m}{2 \pi \hbar\left(t_{b}-t_{a}\right)}\right)^{d / 2} \exp \left[-\frac{m\left(\mathbf{x}_{b}-\mathbf{x}_{a}\right)^{2}}{2 \hbar\left(t_{b}-t_{a}\right)}\right]
$$

or in momentum representation

$$
K\left(\mathbf{x}_{b}-\mathbf{x}_{a} ; t_{b}-t_{a}\right)=\frac{1}{(2 \pi \hbar)^{d}} \int \mathrm{d} \mathbf{p} \exp \left[-\frac{\mathbf{p}^{2}\left(t_{b}-t_{a}\right)}{2 m \hbar}+\frac{\mathrm{i}}{\hbar} \mathbf{p}\left(\mathbf{x}_{b}-\mathbf{x}_{a}\right)\right]
$$

$K\left(\mathbf{x}_{b}-\mathbf{x}_{a} ; t_{b}-t_{a}\right)$ corresponds to the transition amplitude of a free particle for an imaginary time. $K\left(\mathbf{x}_{b}-\mathbf{x}_{a} ; t_{b}-t_{a}\right)$ verifies the group law [8]

$$
K\left(\mathbf{x}_{b}-\mathbf{x}_{a} ; t_{b}-t_{a}\right)=\int K\left(\mathbf{x}_{b}-\mathbf{x}_{c} ; t_{b}-t_{c}\right) K\left(\mathbf{x}_{c}-\mathbf{x}_{a} ; t_{c}-t_{a}\right) \mathrm{d} \mathbf{x}_{c}
$$

and $Z$ is now given by $Z=V K(0 ; \beta \hbar)$. Moreover, it is easy to check that $K\left(\mathbf{x}_{b}-\right.$ $\left.\mathbf{x}_{a} ; t_{b}-t_{a}\right)$ verifies the standard diffusion equation with a diffusion coefficient $\hbar / 2 m$. From $K\left(\mathbf{x}_{b}-\mathbf{x}_{a} ; t_{b}-t_{a}\right)$ we can calculate any average quantity.

If we consider $\beta \hbar$ as an ordinary time, then we can define the velocity as the change of position $\delta \mathbf{x}$ corresponding to a given finite time interval $\delta t$ provided $\delta t \leqslant$ $\beta \hbar$. For a free particle $\langle\delta \mathbf{x} / \delta t\rangle$ vanishes due to the symmetry of space and hereafter we focus on $\left\langle(\delta \mathbf{x} / \delta t)^{2}\right\rangle$. This quantity is given by:

$$
\begin{aligned}
\left\langle\left(\frac{\delta \mathbf{x}}{\delta t}\right)^{2}\right\rangle= & \frac{1}{Z} \int \mathrm{d} \mathbf{x}_{a} d \mathbf{x}_{b} \mathrm{~d} \delta x K\left(\mathbf{x}_{b}-\mathbf{x}_{a} ; t_{b}-0\right) K(\delta \mathbf{x} ; \delta t) \\
& \times\left(\frac{\delta \mathbf{x}}{\delta t}\right)^{2} K\left(\mathbf{x}_{b}-\mathbf{x}_{a}+\delta \mathbf{x} ; \beta \hbar-t_{b}+\delta t\right),
\end{aligned}
$$

from which we can derive the following exact result

$$
\left\langle\left(\frac{\delta \mathbf{x}}{\delta t}\right)^{2}\right\rangle=\left[\frac{\beta \hbar}{\delta t}-1\right] \frac{\left\langle\mathbf{p}^{2}\right\rangle}{m^{2}}=\left[\frac{\beta \hbar}{\delta t}-1\right] \frac{d}{\beta m}
$$

which shows the relation between $\left\langle(\delta \mathbf{x} / \delta t)^{2}\right\rangle$ and $\left\langle\mathbf{p}^{2}\right\rangle$.

In what follows, to be short, we call $K\left(\mathbf{x}_{b}-\mathbf{x}_{a} ; t_{b}-t_{a}\right)$ a propagator although this term is improper since, as shown by (9), it is not sufficient to calculate an average. This is obvious since $K\left(\mathbf{x}_{b}-\mathbf{x}_{a} ; t_{b}-t_{a}\right)$ does not contain the temperature. Due to this, (10) does not correspond to the analytic continuation for an imaginary time of 
the transition amplitude calculated in [3]. A detailed analysis of this point will be given in a subsequent paper.

We can also characterize the path by studying the thermal average of the change of velocity at a given point $\left(\mathbf{x}_{2}, t_{2}\right)$ of the path. In order to do that we consider the quantity $\left\langle\left[\left(\mathbf{x}_{2}-\mathbf{x}_{1}\right) / \delta t\right]\left[\left(\mathbf{x}_{3}-\mathbf{x}_{2}\right) / \delta t\right]\right\rangle$ in which the positions $\mathbf{x}_{1}, \mathbf{x}_{2}$ and $\mathbf{x}_{3}$ are taken at times $t_{1}, t_{2}$ and $t_{3}$ respectively, we must have $0<t_{1}<t_{2}<t_{3}<\beta \hbar$. We can write the average $\left\langle\left[\left(\mathbf{x}_{2}-\mathbf{x}_{1}\right) / \delta t\right]\left[\left(\mathbf{x}_{3}-\mathbf{x}_{2}\right) / \delta t\right]\right\rangle$ in a form similar to equation (9) and using (7) we derive the following exact result

$$
\left\langle\left[\frac{\mathbf{x}_{2}-\mathbf{x}_{1}}{\delta t}\right]\left[\frac{\mathbf{x}_{3}-\mathbf{x}_{2}}{\delta t}\right]\right\rangle=-\frac{\left\langle\mathbf{p}^{2}\right\rangle}{m^{2}}=-\frac{d}{\beta m},
$$

which shows that the path is not differentiable at any time whatever the value of $\delta t$ provided $\delta t<\beta \hbar$. In [6] it has been shown why the quantity calculated in (11) is negative. If the temperature goes to zero the r.h.s. of (11) goes to zero and then, on the trace of the motion, the velocity just before the time $t$ is uncorrelated with the velocity just after this time. At a non-vanishing temperature the role of the thermostat is, in average, to reverse the velocity in agreement with the fact that only closed paths are considered in statistical physics. This induces the negative correlation observed in (11).

The relation (10) shows that $\left\langle(\delta \mathbf{x} / \delta t)^{2}\right\rangle$ grows indefinitely when $\delta t$ goes to zero but from the theory of relativity we know that $\left[\left\langle(\delta \mathbf{x} / \delta t)^{2}\right\rangle\right]^{1 / 2}$ cannot exceed the velocity of light, $c$. Thus we have to start again our calculations in the scheme of special relativity.

\subsection{The relativistic domain}

Due to the special relativity we must change the Hamiltonian by introducing the relativistic dynamics and take into account that new particles can be created. In what follows we will stay in the one-particle formalism and only the change in the Hamiltonian is considered. The details of the calculations which have been given in [6] will be not reproduced here.

For spinless particles, the Klein-Gordon (KG) wave equation is an acceptable starting point for a relativistic approach but the definition of operators in the oneparticle formalism is not a trivial task (see for instance [9-11]). In order to define a meaningful position operator, first we put the KG equation in a Schrödinger form. The price that we have to pay in such a transformation is that the wave function $\psi$ is now a two-component vector. Secondly, we introduce the Feshbach-Villars (FV) representation [10] which transforms $\psi$ into $\varphi$ according to $\varphi=\hat{U} \psi$ where $\hat{U}$ is not a unitary matrix in the usual sense [11]. In this representation, the position operator is defined as usual and its eigenstates of positive and negative energy are given by

$$
\varphi_{x}^{+}(\mathbf{p})=\frac{1}{(2 \pi \hbar)^{d / 2}}\left(\begin{array}{l}
1 \\
0
\end{array}\right) \exp \left(\frac{\mathrm{i}}{\hbar} \mathbf{p} \cdot \mathbf{x}\right), \quad \varphi_{x}^{-}(\mathbf{p})=\frac{1}{(2 \pi \hbar)^{d / 2}}\left(\begin{array}{c}
0 \\
1
\end{array}\right) \exp \left(\frac{\mathrm{i}}{\hbar} \mathbf{p} \cdot \mathbf{x}\right) .
$$

In this $\varphi$-representation we can define the so-called even operators for which there is no mixing between states of positive and negative energy. The expectation $\langle A\rangle$ of 
an operator $A$ is defined according to $[10,11]$

$$
\langle A\rangle=\int \mathrm{d} \mathbf{x} \psi^{\dagger} \tau_{3} A \psi=\int \mathrm{d} \mathbf{x} \varphi^{\dagger} \tau_{3} A_{\varphi} \varphi
$$

in which $\tau_{3}$ is a Pauli matrix (for definition see for instance $[10,11]$ ) and $A_{\varphi}=\hat{U} A \hat{U}^{-1}$. By analogy with (13), we suggest to define the partition function as:

$$
Z=\frac{1}{(2 \pi \hbar)^{d}} \int \mathrm{d} \mathbf{p} \mathrm{d} \mathbf{x} \psi_{x}^{\dagger}(\mathbf{p}) \tau_{3} \exp \left(-\beta H_{\psi}(\mathbf{p})\right) \psi_{x}(\mathbf{p})
$$

in which $H_{\psi}$ is a $2 \times 2$ matrix which contains a combination of the momentum operators and $m c^{2}$ via the Pauli matrices. In FV representation we have

$$
Z=\frac{1}{(2 \pi \hbar)^{d}} \int \mathrm{d} \mathbf{p d} \mathbf{x} \varphi_{x}^{\dagger}(\mathbf{p}) \tau_{3} \exp \left(-\beta H_{\varphi}(\mathbf{p})\right) \varphi_{x}(\mathbf{p})
$$

in which the Hamiltonian is a $2 \times 2$ matrix given by

$$
\beta H_{\varphi}(\mathbf{p})=\tau_{3} \sigma \sqrt{1+\left(\frac{\mathbf{p}}{m c}\right)^{2}}, \quad \sigma=\beta m c^{2}
$$

and $\varphi_{x}(\mathbf{p})$ corresponds to $\varphi_{x}^{+}(\mathbf{p})$ given by (12). Using the properties of the matrix $\tau_{3}$, it is easy to see that $Z$ is given by

$$
Z=\frac{V}{(2 \pi \hbar)^{d}} \int \mathrm{d} \mathbf{p} \exp \left[-\sigma\left(\sqrt{1+\left(\frac{\mathbf{p}}{m c}\right)^{2}}\right)\right]
$$

which is a natural extension of (3). Such an expression corresponds to $1 /(2 \pi \hbar)^{d}$ times the classical partition function introduced by Pauli [12]. In the FV representation we define a propagator according to

$$
K\left(\mathbf{x}_{b}-\mathbf{x}_{a} ; t_{b}-t_{a}\right)=\int \mathrm{d} \mathbf{p} \varphi_{x a}^{\dagger}(\mathbf{p}) \tau_{3} \exp \left[-\left(t_{b}-t_{a}\right) H_{\varphi}(\mathbf{p}) / \hbar\right] \varphi_{x b}(\mathbf{p})
$$

Using the explicit expression of $\varphi_{x}(\mathbf{p})$ and the properties of $\tau_{3}$, we can write (17) as

$$
K\left(\mathbf{x}_{b}-\mathbf{x}_{a} ; t_{b}-t_{a}\right)=\int \frac{\mathrm{d} \mathbf{p}}{E_{p}} \frac{1}{(2 \pi \hbar)^{d / 2}} \mathrm{e}^{\mathrm{i} \mathbf{p} \cdot \mathbf{x}_{b} / \hbar} E_{p}^{1 / 2} \frac{1}{(2 \pi \hbar)^{d / 2}} \mathrm{e}^{\mathrm{i} \mathbf{p} \cdot \mathbf{x}_{a} / \hbar} E_{p}^{1 / 2} \mathrm{e}^{-\left(t_{b}-t_{a}\right) E_{p} / \hbar}
$$

where $E_{p}=\left[\mathbf{p}^{2} c^{2}+m^{2} c^{4}\right]^{1 / 2}$ and we have isolated the Lorentz invariant measure $\left[\mathrm{d} \mathbf{p} / E_{p}\right]$. In (18), the quantity $\left(E_{p}^{1 / 2} /(2 \pi \hbar)^{d / 2}\right) \mathrm{e}^{\mathbf{i} \mathbf{p} \cdot \mathbf{x}_{b} / \hbar}$ is, in $\mathbf{p}$-representation, the exact form for a state localized at $\mathbf{x}_{b}$ for $t=0$ in the relativistic domain; this result has been derived by Newton and Wigner from first principle arguments [9]. It shows that a localized state has a spatial extension given by the Compton wave length $\lambda=\hbar / m c$. The propagator (18) verifies the composition rule (8) and we have $Z=V K(0 ; \beta \hbar)$. In terms of the dimensionless quantities $\mathbf{u}=\mathbf{p} /(m c) ; \quad \tau=$ 
$t /(\beta \hbar) ; \quad \mathbf{r}=\mathbf{x} / \lambda . K\left(\mathbf{x}_{b}-\mathbf{x}_{a} ; t_{b}-t_{a}\right)$ becomes $K\left(\mathbf{r}_{b}-\mathbf{r}_{a} ; \tau_{b}-\tau_{a}\right)$ and takes the explicit form in the case $d=1$

$$
K\left(\mathbf{r}_{b}-\mathbf{r}_{a} ; \tau_{b}-\tau_{a}\right)=\frac{1}{\pi \lambda} \frac{\sigma\left(\tau_{b}-\tau_{a}\right)}{\sqrt{\sigma^{2}\left(\tau_{b}-\tau_{a}\right)^{2}+\left(\mathbf{r}_{b}-\mathbf{r}_{a}\right)^{2}}} K_{1}\left(\sqrt{\sigma^{2}\left(\tau_{b}-\tau_{a}\right)^{2}+\left(\mathbf{r}_{b}-\mathbf{r}_{a}\right)^{2}}\right),
$$

where $K_{1}(z)$ is a modified Bessel function. A similar result is obtained in the case $d=3$. In the limit $c$ goes to infinite, (19) becomes $\exp \left[-m c^{2}\left(t_{b}-t_{a}\right) / \hbar\right]$ times the non-relativistic propagator (6). In order to investigate the quantum dynamics of a relativistic free particle a propagator similar to (18) has been considered in [13], it corresponds to (18) in the real-imaginary time transformation.

Starting from the propagator (18) and after some straightforward integrations we get the following exact results:

$$
\left\langle\left(\frac{\delta \mathbf{x}}{\delta t}\right)^{2}\right\rangle=\left[\frac{\beta \hbar}{\delta t}-1\right]\left\langle\frac{\mathbf{p}^{2} c^{4}}{\mathbf{p}^{2} c^{2}+m^{2} c^{4}}\right\rangle
$$

and

$$
\left\langle\frac{\mathbf{x}_{2}-\mathbf{x}_{1}}{\delta t} \cdot \frac{\mathbf{x}_{3}-\mathbf{x}_{2}}{\delta t}\right\rangle=-\left\langle\frac{\mathbf{p}^{2} c^{4}}{\mathbf{p}^{2} c^{2}+m^{2} c^{4}}\right\rangle .
$$

The equations (20) and (21) represent the generalization of (10) and (11) to the relativistic domain. As in the non-relativistic case (20) and (21) are not a simple analytic continuation for imaginary time of the transition elements calculated in [3].

Recently, H.Kleinert [7] introduced a propagator associated with the KG equation. This propagator can be put in a form similar to (18), for $\mathrm{d}=1$ it is given by

$$
K^{\prime}\left(\mathbf{x}_{b}-\mathbf{x}_{a} ; t_{b}-t_{a}\right)=\int \frac{\mathrm{d} \mathbf{p}}{E_{p}} \frac{1}{(2 \pi \hbar)^{-d / 2}} \mathrm{e}^{\mathrm{i} \mathbf{p} \cdot \mathbf{x}_{\mathbf{b}} / \hbar} \frac{1}{(2 \pi \hbar)^{-d / 2}} \mathrm{e}^{\mathrm{i} \mathbf{p} \cdot \mathbf{x}_{\mathbf{a}} / \hbar} \mathrm{e}^{-\left(t_{b}-t_{a}\right) E_{p} / \hbar}
$$

that we can also write as

$$
K^{\prime}\left(\mathbf{r}_{b}-\mathbf{r}_{a} ; \tau_{b}-\tau_{a}\right)=\frac{1}{2 \pi \lambda} K_{0}\left(\sqrt{\sigma^{2}\left(\tau_{b}-\tau_{a}\right)^{2}+\left(\mathbf{r}_{b}-\mathbf{r}_{a}\right)^{2}}\right)
$$

which is clearly in disagreement with our result (19). The origin of the discrepancy is related to the fact that in (22) we assume that the localized states correspond to a delta function in real space contrary to what has been established in [9]. The use of (23) instead of (19) leads to the results which have no clear physical meaning. In particular, we do not find (16) the expected classical limit.

When $\delta t \ll \beta \hbar$ and, $d=1$, we get from (20)

$$
\left\langle\left(\frac{\delta \mathbf{x}}{\delta t}\right)^{2}\right\rangle \sim \frac{\hbar}{m \delta t} \frac{\int \mathrm{d} \mathbf{u}\left(\mathbf{u}^{2}+1\right)^{-3 / 2} \exp \left[-\sigma\left(\mathbf{u}^{2}+1\right)^{1 / 2}\right]}{\int \mathrm{d} \mathbf{u} \exp \left[-\sigma\left(\mathbf{u}^{2}+1\right)^{1 / 2}\right]} .
$$

A similar result is obtained for $d=3$, only the ratio of integrals in the r.h.s. of (24) is different. In general we can write $\left\langle(\delta \mathbf{x} / \delta t)^{2}\right\rangle / c^{2}=\hbar /\left(m c^{2} \delta t\right) f(\sigma)$ where $f(\sigma)$ is 
approximately one for large values of $\sigma$. Thus, for $\delta t \ll \beta \hbar$ we get $\left\langle(\delta \mathbf{x})^{2}\right\rangle=\frac{\hbar}{m} \delta t$. Since $\left\langle(\delta \mathbf{x} / \delta t)^{2}\right\rangle / c^{2}$ must be smaller than one, the time interval $\delta t$ must be such as $\delta t \geqslant \hbar / m c^{2}$ which is an expected result since we are in a regime with a fixed number of particles. For a smaller time interval the uncertainty relation $\delta t \delta E=\hbar$ shows that we could create new particles. Thus $\delta t$ must be large enough to avoid the creation of particles by quantum fluctuations. This constraint on $\delta t$ implies that we must have $\beta \hbar \geqslant \delta t \geqslant \hbar / m c^{2}$ leading to $\sigma=\beta m c^{2} \geqslant 1$ which means that no particle can be created by thermal excitation. Thus the relation (20) is exact in the one-particle formalism.

For the smallest time interval $\delta t=\hbar /\left(m c^{2}\right)$ we get the smallest value of $\left\langle(\delta \mathbf{x})^{2}\right\rangle$ :

$$
\left\langle(\delta \mathbf{x})^{2}\right\rangle=\lambda^{2}\left(\beta m c^{2}-1\right)\left\langle\frac{\mathbf{p}^{2} c^{2}}{\mathbf{p}^{2} c^{2}+m^{2} c^{4}}\right\rangle
$$

which shows the interplay between the Compton wavelength, $\lambda$, and the properties of the thermostat. At a very low temperature $\left\langle(\delta \mathbf{x})^{2}\right\rangle$ tends to $\lambda^{2}$, as expected. In addition the largest value of $\left\langle(\delta \mathbf{x})^{2}\right\rangle$ corresponds to

$$
\left\langle(\delta \mathbf{x})^{2}\right\rangle=\left(\frac{\beta \hbar}{2}\right)^{2}\left\langle\frac{\mathbf{p}^{2} c^{4}}{\mathbf{p}^{2} c^{2}+m^{2} c^{4}}\right\rangle .
$$

For large values of $\sigma$ this quantity is the square of the thermal de Broglie wavelength $\Lambda=2 \pi \beta \hbar^{2} / m$. Thus with the path integral formalism, at the thermal equilibrium, we explore some distances larger than $\lambda$ but smaller than $\Lambda$, this is also an expected result. We can note that (20) does not lead to the pure relativistic regime for which we expect $\left\langle(\delta \mathbf{x})^{2}\right\rangle \sim(c \delta t)^{2}$.

\section{Analysis of the results}

The comparison of (20) and (21) with (10) and (11) shows that the quantum paths have similar properties in non-relativistic and relativistic cases provided we stay in the one-particle formalism. This first extension was needed in order to show the that fractal behaviour is something general.

\subsection{Fractal character of the paths}

In statistical physics the paths are closed and due to this the particles do not perform the standard Brownian motion. The relations (21) and (11) show that, in average, there is no derivative on the paths but (20) and (10) do not correspond to the relation between $\left\langle(\delta \mathbf{x})^{2}\right\rangle$ and $\delta t$ which exists in the Brownian motion. In order to recover this relation we have to take the limit $\delta t \ll \beta \hbar$. In [6] an analysis of the quantum paths has been performed in the pure quantum domain i.e. starting from the time dependent Schrödinger equation or from the KG equation. This leads to the investigation of the evolution of a Gaussian wave packet. At $t=0$ the particles are located near the origin, $\mathbf{x}=\mathbf{0}$, with a given initial spatial resolution, $\delta$, and we 
study how $\left\langle(\delta \mathbf{x})^{2}\right\rangle$ depends both on $t$ and $\delta$. In the relativistic domain we must have $\delta \geqslant \lambda$. In the limit $\delta \gg \lambda$ we get [6] the following result

$$
\left\langle(\delta \mathbf{x})^{2}\right\rangle=\frac{\delta^{2}}{2}+\frac{(c t)^{2}}{2}\left(\frac{\lambda}{\delta}\right)^{2}=\frac{\delta^{2}}{2}+\frac{1}{2}\left(\frac{\hbar t}{m}\right)^{2} \frac{1}{\delta^{2}},
$$

provided $\lambda c t \gg \delta^{2} \gg \lambda^{2}$ or $m c^{2} t \gg \hbar$ which is also the condition obtained above in order to observe a Brownian motion in the relativistic domain when the path integral formalism is used. For time intervals, $\delta t$, such as $\hbar t / m \gg \delta^{2}$ we see that $\left\langle(\delta \mathbf{x})^{2}\right\rangle^{1 / 2}$ behaves like $1 / \delta$ leading to a Hausdorff dimension $D_{\mathrm{H}}=2$ as already established in $[9,17]$ (for a definition of $D_{\mathrm{H}}$ see for instance $[2]$ ). Note that the spatial resolution $\delta$ leads to a time resolution $\delta t$. We may define $\delta t$ as the shorter time interval we need to wait in order to see something i.e. to see a displacement of order $\delta$. From the result given above this leads to $\frac{1}{2}(\hbar \delta t / m)^{2} 1 / \delta^{2} \sim \delta^{2}$ or $\hbar \delta t / m=\delta^{2}$. This result is reminiscent of the one obtained above in the path integral formalism. It can be interpreted as follows: $\delta t$ is the time interval for which the particle may explore by diffusion a distance equal to the spatial resolution.

\subsection{Fluctuations of energy}

The interpretation of the results is simple if we give to $\beta \hbar$ the meaning of an ordinary time interval, i.e. a quantity that we can measure using a clock. Then (10) and (20) relate the average of the square of the velocity to its standard expression in terms of momentum, however this relation is not the usual one since we have an extra term $[\beta \hbar / \delta t-1]$ which contains a mixture of quantum effects $(\hbar)$ and thermal effects $(\beta)$. The thermal effects disappear in the limit $\delta t \ll \beta \hbar$, then only the Planck constant remains in the formula.

Using the constrains on the time interval $\hbar / m c^{2} \leqslant \delta t \leqslant \beta \hbar$ we have seen in the previous Section that the particle explores a volume located between two concentric spheres. The radius of the smallest one is the Compton wavelength $(\lambda)$ while the radius of the largest one corresponds to the thermal de Broglie wavelength $(\Lambda)$. We can show that the paths correspond to fluctuating trajectories for which the particles do not verify the equations of motion. To illustrate this point we start from (4) which shows that the lagrangian, $L$, of the particle is given by $L=\frac{1}{2} m[\dot{\mathbf{x}}(t)]^{2}$. From the Euler-Lagrange equations and the cyclic condition on the trajectory $(\mathbf{x}(0)=\mathbf{x}(\beta \hbar))$, we conclude that the particle does not move and stays at the origin of the coordinates. This result which corresponds to a mean field approach can not represent the real physics because, in this case the particle should have a perfectly known position and its momentum should be totally undetermined. Not to violate the basis of quantum physics requires to consider that the particle moves on trajectories which do not verify the equations of motion. This point illustrates the following important aspect of the path integral formalism in statistical physics.

In the classical textbooks on statistical physics [14] we first establish the form of the micro-canonical density matrix $\rho$ and then we put $\rho$ in its canonical form. After that, using the Lie-Trotter formula [7] we may derive (1). Obviously, the validity of 
this derivation is primarily based on the constraints associated with the existence of the micro-canonical expression for $\rho$. In order to define the canonical form of $\rho$ we avoid large quantum fluctuations of energy $\widetilde{\delta E}$ and thus from the time-energy uncertainty relation we must restrict our investigations to time interval $\widetilde{\delta} t$ which are large enough. In (1) no such restriction appears explicitly, for instance in the non-relativistic case we performed an integration from $t=0$ to $t=\beta \hbar$ and the results are meaningful whatever the value of $\delta t$. However, we can note that a similar time-energy uncertainty relation appears at the end of the calculation since we can rewrite (20) according to

$$
\delta t \frac{m}{2}\left\langle\left(\frac{\delta \mathbf{x}}{\delta t}\right)^{2}\right\rangle=\frac{m}{2}(\beta \hbar-\delta t)\left\langle\frac{\mathbf{p}^{2} c^{4}}{\mathbf{p}^{2} c^{2}+m^{2} c^{4}}\right\rangle
$$

in which the r.h.s. tends to $\hbar$ if $\delta t \ll \beta \hbar$ and $\sigma \gg 1$. It is interesting to note that $m / 2\left\langle(\delta \mathbf{x} / \delta t)^{2}\right\rangle$ is not the kinetic energy, $U$, in the thermodynamic sense. This quantity is defined according to $U=-\partial \ln Z / \partial \beta$ and its value is $(d / 2 \beta)$, for instance in the non-relativistic case. The quantity $m / 2\left\langle(\delta \mathbf{x} / \delta t)^{2}\right\rangle$ represents a fluctuating energy associated with the fact that, on the path integral formalism, the particles do not follow the equation of motion and then the energy is not constant along the quantum path as noted in [15]. Thus, we are led to consider that (1) is not strictly equivalent to the canonical formalism derived by the usual routes but represents a bit more general result as suggested by Feynman [3].

In what follows based on the general arguments taken outside the path integral formalism, we will try to see what kind of physics is expected when an ordinary time interval becomes smaller than the quantity $\beta \hbar$ which has the same dimension as a time. In order to clarify the discussion, the ordinary time interval (that we can measure using a clock) will be noted $\tau$ to avoid the confusion with $\delta t$ introduced above.

\subsection{Thermal and quantum fluctuations}

In standard statistical thermodynamics it is assumed that the quantum fluctuations are negligible compared to the thermal fluctuations. This is quite clear in usual textbooks where the energy conservation law is expected to hold between the system under investigation and the reservoir [14]. Now, let us consider that a quantum fluctuation of energy, $\delta E$, takes place during a time interval $\tau$. The uncertainty relation leads to $\tau \delta E \geqslant \hbar$. In order to have $\beta \delta E \leqslant 1$ we must have $\tau \geqslant \beta \hbar$. Thus, when investigating the domain $\tau \leqslant \beta \hbar$ it seems quite natural to observe a very intricate mixture between quantum physics and thermodynamics. If we consider that $\tau$ corresponds to the $\delta t$ investigated above, it is very tempting to assume that $(20)$ and (10) give this complicated physics. Moreover from the uncertainty relation it is clear that $\delta E$ must behave like $1 / \tau$, of course this is what we find if $E$ is associated with the kinetic energy on the path. Thus, we see that thermostat fixes the average value of the momentum and the usual thermodynamics but not the velocity which 
also depends on the time interval " $\delta t$ " and the Planck constant. Note that $\beta \hbar$ corresponds to 10 femtoseconds at the room temperature, a domain that we can explore nowadays using sophisticated lasers.

\subsection{Relativistic invariance and thermodynamics}

Let us associate to the system under investigation a given frame in which there is a clock located at the origin of the coordinates. In this frame we measure a time interval $\tau$ and a temperature $T$. This system moves with a uniform velocity relative to another frame where we measure a time interval $\tau^{\prime}$ and $T^{\prime}$. If we accept the usual law of transformation for $T$ given for instance in [12] then we observe that the product $T \tau$ must be Lorentz invariant. Thus, in order to elaborate a statistical physics in which the covariance should be manifest, it seems natural to characterize the thermostat by a variable like $T \tau$ rather than just by $T$. In the path integral formalism if we consider $\beta \hbar$ as an ordinary time interval $\tau$ then we have $T \tau=\hbar / k_{\mathrm{B}}$. The product $T \tau$ is not only manifestly Lorentz invariant but we know its value, $\hbar / k_{\mathrm{B}}$.

Let us recall that the main goal of this paper is to investigate if the fractal character which appears via the path integral formalism is something fundamental or just a useful mathematical trick. A similar question has been considered concerning the Carnot's principle or the second principle of thermodynamics (for a summary in this field see [16]). To elucidate this question we have to deal with the famous Maxwell's demon. After a careful analysis, Brillouin [16] has shown that the demon cannot operate proving the fundamental nature of the Carnot's principle. The main point of the Brillouin's analysis is the following: in order to see the molecules in the vessel, the demon needs a source of light which can be distinguished from the background of black-body radiation which exists in the vessel. Let be $T$ the temperature of the vessel and $\tau$ the period of the source of light. If $T \tau$ is larger than $\hbar / k_{\mathrm{B}}$ the demon can not get any information and then he can not violate the second principle, otherwise he can separate the molecules but the Carnot's principle is verified. Thus the ratio $\hbar / k_{\mathrm{B}}$ appears as a critical parameter which determines the domain in which we can verify that the Carnot's principle has a fundamental nature and the time interval at which we can observe the fractal nature of the quantum paths in statistical physics. From these elements we may conjecture that the ratio $\hbar / k_{\mathrm{B}}$ is probably a fundamental quantity in the foundation of a covariant statistical physics.

\section{Conclusions}

Using the path integral formalism of statistical physics we have investigated the behaviour of an ideal gas of spinless particles. If we focus on the thermodynamics or on the properties related to the momentum, we recover the standard results including their extension to the relativistic domain. The temperature which appears in the partition function fixes the pressure or the kinetic energy but not all the quantities in the system. The thermostat does not fix the average value of the velocity on the path 
which depends on the time interval on which we investigate the motion of particles. The particles do not follow the equation of motion but their stochastic behaviour does not strictly correspond to the Brownian motion since only the closed paths are considered. For time intervals much shorter than $\beta \hbar$ the standard properties of the Brownian motion are recovered. All the results are exact provided we stay in the one-particle formalism. This requires working on the time interval larger than $\hbar / m c^{2}$ and on the distances larger than the Compton wavelength.

If we consider $\beta \hbar$ as an ordinary time, all the results have a simple and meaningful interpretation. For instance, during their motion the particles explore a spherical volume of radius corresponding to the thermal de Broglie wavelength and on the paths we may consider a time-energy uncertainty relation which has its usual meaning. More fundamentally we have established a link between the domain in which we can see the existence of a fractal behaviour and the domain on which we can test the Carnot's principle. These two phenomena are conditioned by the ratio $\hbar / k_{\mathrm{B}}$. This point requires further investigations and suggests that this ratio may play a fundamental role in the foundation of covariant statistical physics. It is interesting to note that $\beta \hbar$ is about 10 femtoseconds at the room temperature. For this time scale we are beyond the thermal physics since thermal and quantum fluctuations have the same order of magnitude and new physics can be expected.

\section{Acknowledgements}

The author wants to thank Dr. A. Le Méhauté for very exciting discussions regarding this field of investigations.

\section{References}

1. Addison P. - In: Fractals and Chaos. Philadelphia, Institue of Physics Publishing, 1997.

2. Mandelbrot B. - In: Fractals. San Francisco, Freeman, 1977.

3. Feynman R.P., Hibbs A.R. - In: Quantum Mechanics and Path Integrals. Mc Graw Hill, New York, 1965.

4. Kroger H. // Physics Report, 2000, vol. 323, p. 81.

5. Le Méhauté A., Nigmatullin R., Nivanen L. - In: Geometry and Physics of Fractional Calculus. Paris, Hermes Editions (in press).

6. Badiali J.P. // Phys. Rev. E, 1999, vol. 60, p. 2533.

7. Kleinert H. - In: Path Integrals in Quantum Mechanics, Statistics and Polymer Physics. Singapore, World Scientific, 1995.

8. Dittrich W., Reuter M. - In: Classical and Quantum Dynamics. Berlin, Heidelberg, Springer Verlag, 1992.

9. Newton T.D., Wigner E.P. // Rev. Mod. Phys., 1949, vol. 21, p. 400.

10. Feshbach H., Villars F. // Rev. Mod. Phys., 1958, vol. 30, p. 24.

11. Greiner W. - In: Relativistic Quantum Mechanics. 2nd edition. Berlin, Springer, 1997.

12. Pauli W. Theory of Relativity. New York, Dover, 1958.

13. Hartle J.B., Kuchar K.V. // Phys. Rev. D, 1986, vol. 34, p. 2323. 
14. Tolman R.C. - In: The Principles of Statistical Mechanics. New York, Dover, 1979.

15. Weinberg S. - In: The Quantum Theory of Fields. Cambridge, Cambridge University Press, 1995.

16. Brillouin L. Science and Information Theory. New York, Academic Press, 1962.

17. Abbott L.F., Wise M.B. // Am. J. Phys., 1981, vol. 49, p. 37.

18. Nottale L. - In: Fractal Space-time and Microphysics. Singapore, World Scientific Publishing, 1993.

19. Cannata F., Ferrari L. // Am. J. Phys., 1988, vol. 56, p. 8. 


\title{
Фрактальна поведінка квантових шляхів у статистичній фізиці
}

\author{
Ж.П.Бадіалі \\ Лабораторія структури та реактивності поверхні, \\ Університет ім. П'єра і Марії Кюрі, \\ Франція, 75230 Париж, Площа Жюсс'ї
}

Отримано 29 травня 2000 р.

Використовується формалізм інтегралів по шляхах для опису ідеального газу безспінових частинок. Показано, що квантові шляхи мають однакові властивості у релятивістичній та нерелятивістичній областях, за умови, що немає утворення нових частинок. Введено деякі величини, пов'язані зі шляхами. Ці величини мають просте значення, якщо величина $\beta \hbar$, де $\beta$ - обернена температура, розглядається як звичайний час. Співвідношення між швидкістю на шляху та імпульсом не є звичним, появляється додатковий член, який показує, що термостат не може фіксувати середнє значення цієї швидкості. Хоча усі термодинамічні величини приймають традиційні значення.

Шляхи описують флуктуаційні траєкторії, на яких частинки підкоряються рівнянням руху. Для інтервалів часу, значно коротших за $\beta \hbar$, ми відтворюємо властивості Броунівського руху. Траєкторії є обмежені у просторі між об'ємом порядку комптонівської довжини хвилі для коротких відстаней та об'ємом порядку температурної довжини хвилі де Бройля для найбільших відстаней. Показано, що невизначеність між часом та енергією справджується на квантових шляхах. Це вказує на те, що матриця густини, отримана квантуванням класичної канонічної функції розподілу, не має бути цілком еквівалентною до матриці густини, отриманої звиклим способом. Наведено переконливі аргументи на користь того, що $\beta \hbar$ може розглядатись як звичайний час, а не як формальна величина, що має розмірність часу. Ця стаття показує, що для інтервалів часу порядку 10 фемтосекунд, можна очікувати цілком нову фізику при кімнатній температурі. Також ці результати наводять на думку, що відношення $\hbar / k_{b}$ може відігравати вирішальну роль у побудові коваріантної статистичної фізики.

Ключові слова: інтеграл по шляхах, статистична фізика, флуктуації, відносність

PACS: 03.65.Ca, 05.30. $-d, 05.70 .-a, 47.53 .+n$ 